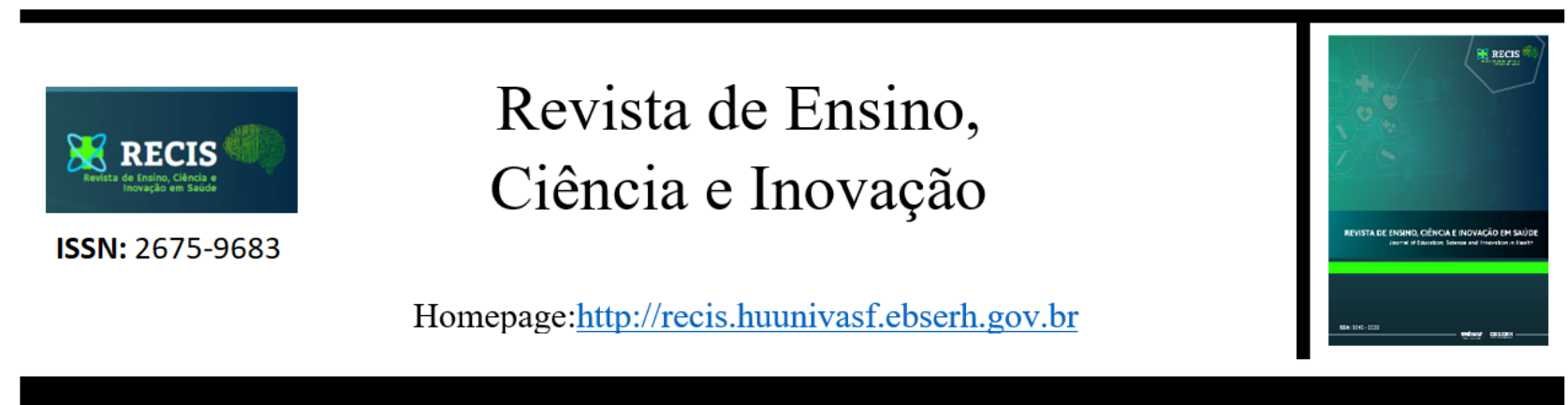

\title{
Redes Colaborativas Brasileiras em Qualidade de Vida: Análise Cientométrica das publicações de 2000 a 2011
}

\section{Brazilian Collaborative Networks in Quality of Life: Scientometric analysis of publications from 2000 to 2011}

\author{
André Luis Mezzadri' ${ }^{1}$, Bruno Pedroso², Luiz Alberto Pilatti ${ }^{1}$, Guilherme Moreira Caetano \\ Pinto $^{2}$, Claudia Tania Picinin ${ }^{1}$
}

${ }^{1}$ Universidade Tecnológica Federal do Paraná, ${ }^{2}$ Universidade Estadual de Ponta Grossa.

Autor correspondente: claudiapicinin@utfpr.edu.br

Artigo recebido em 01/02/2021 e aceito em 19/04/2021-

\begin{abstract}
RESUMO
O presente estudo tem por objetivo identificar as redes em trabalhos científicos com escopo na área de Qualidade de Vida (QV) publicados em periódicos indexados na base de dados Scientific Electronic Library Online (Scielo) Brasil no período de 2000 a 2011. A coleta de dados ocorreu entre junho e setembro de 2013, e foi realizada na Universidade Tecnológica Federal do Paraná do município de Ponta Grossa, localizado no estado do Paraná. Foi utilizado o unitermo "Qualidade de Vida", "Qualidade de Vida Relacionada à Saúde" e "Qualidade de Vida no Trabalho" (QVT). O número de autores totalizou 2.529. Estes pesquisadores estão afiliados a 328 instituições distintas, das quais a maioria são Instituições de Ensino Superior, hospitais, centros de pesquisa, clínicas ou instituições parceiras ao setor de pós-graduação, pesquisa e extensão. No período totalizou-se 3.192 publicações em QV em 100 periódicos distintos, sendo que dos 3.192 artigos, 3.152 foram indexados com o unitermo "qualidade de vida", 21 foram indexados com o unitermo "qualidade de vida relacionada à saúde" e 19 foram indexados com o unitermo "qualidade de vida no trabalho". Conclui-se que os temas QV e QVT passaram a ser estudados por inúmeras áreas do conhecimento, pois inicialmente eram objeto de análise principalmente da sociologia e administração.

Palavras-Chave: Qualidade de Vida; Bibliometria; Colaboração Intersetorial.
\end{abstract}

\begin{abstract}
The present study has the objective identifier the scientific work web with scope in the field about Quality of Life (LQ) published in indexed journals in Scielo Brasil data base from 2000 to 2011. Data collection was carried out between June and September 2013, at the Federal Technological University of Paraná in the municipality of Ponta Grossa, located in the state of Paraná.It was used the keywords "Quality of Life', "quality of life related to health" and "Quality of Working Life" (QWL). The number of authors totalized 2,529. These researchers are affiliated to 328 distinct institutions, that the majority are Higher Education Institutions (HEIs), hospitals, research center, clinics or partner institutions to the post-graduation, research and extension. During the period, amounted to 3,192 publishes in QOL in 100 distinct journals, of the total 3,192 papers, 3,152 were indexed with keyword "Quality of Life", 21 were indexed with keyword "quality of life related to health" and 19 with the keyword "quality of working life". From the foregoing, it is apparent that the QOL and QWL issues were initially only analysis objects of sociology and management and that at present, have been studies by many other areas of knowledge. Keywords: Quality of Life; Bibliometrics; Intesectorial Collaboration.
\end{abstract}




\section{INTRODUÇÃO}

A produção científica do Brasil encontra-se em desenvolvimento ${ }^{1}$. Face a este contexto, destaca-se o crescimento de estudos cientométricos $^{2}$. As pesquisas de cunho bibliométrico e/ou cientométrico tem por objetivo investigar a produção científica sob diferentes perspectivas, e permitem identificar e analisar a atividade científica de pesquisadores $3,4,5,6,7$. A Bibliometria aborda aspectos quantitativos e, por sua vez, a Cientometria analisa os aspectos quantitativos que tem relação com a produção do conhecimento ${ }^{3}$.

Dentre as pesquisas cientométricas tem-se a investigação sobre as redes de colaboração em trabalhos científicos. Desde o século XIX são registrados esforços colaborativos envolvendo pesquisadores. $^{8}$

As colaborações entre os pesquisadores estão ligadas as redes sociais, que originadas do relacionamento entre os indivíduos inseridos em um mesmo ambiente, oportunizam o fortalecimento das atividades de pesquisa e produção científica de forma coletiva através das coautorias ${ }^{9}$. Neste sentido, as pesquisas sobre as redes são utilizadas para a compreensão de diferentes cenários, evidenciando problemas relacionados a produção científica.

Paker $^{10}$ aponta alguns problemas na produção científica brasileira, especialmente em relação a internacionalização dos periódicos nacionais. $\mathrm{O}$ autor indica que o predomínio de autores brasileiros, a baixa porcentagem de artigos em colaboração internacional, o predomínio do português nas publicações, as composições dos conselhos editorais, os processos de revisão dos manuscritos com baixíssimos níveis de profissionalização, e a sustentabilidade financeira dificultam o processo de internacionalização dos periódicos brasileiros.

Os periódicos brasileiros, em sua grande parte, estão no indexador Scientific Electronic Library Online (Scielo). Criado em 1997 por Rogério Meneghini e Abel Packer, a Scielo proporcionou concentração e visibilidade para centenas de periódicos brasileiros de acesso aberto, sendo considerado em meados de 2013 o maior indexador da América Latina, proporcionando controle de qualidade do conteúdo oferecido por 1.166 periódicos de 20 países. $^{10}$
Com o objetivo de "contribuir para melhorar a qualidade, o acesso e o impacto dos periódicos por meio da indexação e publicação online na web em acesso aberto", a Scielo permitiu à inserção de conteúdos locais e regionais no fluxo global de informação científica, com o escoamento de artigos de autores brasileiros que não encontravam guarida em periódicos internacionais, por não apresentarem qualidade suficiente ou por tratarem de assuntos muito locais. ${ }^{10,11}$

Apesar da Scielo, os periódicos nacionais não deixaram de ser a segunda opção de publicação dos 'bons pesquisadores', produtores de artigos de qualidade superior, que buscam serem lidos e citados. ${ }^{11}$ É fato que os periódicos nacionais, mesmo os de maior visibilidade, apresentam menor número de citações de seus artigos e fatores de impacto inferiores comparados com periódicos similares de países desenvolvidos.

A Scielo fez com que os periódicos e os artigos nacionais tivessem suporte e melhorassem. No entanto, apesar da boa visibilidade internacional, chegou-se em um momento em que, com o sistema atual, melhorar a qualidade se tornou totalmente inviável, sendo necessária uma mudança significativa. ${ }^{11}$ Neste sentido, o aumento do desempenho internacional dos periódicos brasileiros em termos de citações que recebem por artigo é o desafio da Scielo, no qual as pesquisas sobre as redes de colaboração podem auxiliar.

A produção em rede tem sido estudada em abordagem acerca dos periódicos nacionais e internacionais ${ }^{12}$, sobre os indicadores de coautoria $^{13,14}$, quanto a caracterização das redes e sua relação com o aumento da produção científica ${ }^{1,15,16}$, e/ou em diferentes áreas do conhecimento, tais como as ciências contábeis ${ }^{9}$, administração pública da saúde $^{17}$, ciência e saúde coletiva ${ }^{18}$, política educacional no Brasil ${ }^{19}$.

Neste sentido, com as possibilidades advindas das Tecnologias de Informação e de Comunicação (TICs) e as redes sociais de colaboração, as formas de se fazer ciência foram, e continuam sendo, definitivamente transmudas.

Face as contínuas alterações na ciência e as especificidades torna-se interessante investigações em diferentes áreas do conhecimento, tais como a área da Qualidade de Vida. Neste sentido, apresentase as seguintes perguntas de partida: Quais são as redes existentes em trabalhos científicos com escopo na Qualidade de vida publicados em periódicos indexados na Scielo Brasil? Quais as áreas de conhecimento e instituições de ensino superior 
concentram o maior número de publicações sobre qualidade de vida nos periódicos indexados na base de dados Scielo Brasil?

A resolução do presente problema de pesquisa justifica-se pela qualidade de vida tratar-se de um tema contemporâneo, subjetivo, complexo e multidimensional, constituído por fatores intrínsecos (alterações fisiológicas) e extrínsecos (aspectos sociais), e com característica multidisciplinar.

Face ao exposto, o presente estudo tem por objetivo identificar as redes em trabalhos científicos com escopo na qualidade de vida publicados, no período de 2000 a 2011, em periódicos indexados na Scielo Brasil. Tem-se como hipótese: os pesquisadores da área da medicina apresentam maior número de publicações sobre Qualidade de vida; as instituições públicas apresentam maior número de pesquisas sobre qualidade de vida em periódicos indexados na Scielo.

\section{ANÁLISE DE REDES SOCIAIS}

As redes sociais "influenciam o comportamento de indivíduos e grupos" ${ }^{20}$. Para Souza e Quandt ${ }^{21}$, a análise das redes possui origem interdisciplinar e "o objetivo da metodologia é realizar o levantamento de propriedades e conteúdos provenientes da interação entre unidades interdependentes", sendo que a análise de redes sociais evita o reducionismo metodológico. Análise de redes sociais pode ser aplicada a fenômenos de larga escala. ${ }^{22}$

O princípio básico da análise de redes é que "a estrutura das relações sociais determina o conteúdo dessas relações". ${ }^{20}$ Nesse contexto, o principal conteúdo da vida social inclui as organizações, mas não limita-se às suas fronteiras, representando assim as relações sociais.

Castells ${ }^{23}$ alerta para o fenômeno das redes como uma nova morfologia social que altera profundamente os fluxos de informação, a cultura e os modos de produção. O poder dos fluxos de informação, em especial possibilitado pelas novas tecnologias, passa a exercer um papel mais importante que os próprios fluxos de poder. ${ }^{5}$

Para Mizruchi ${ }^{20}$, três áreas teóricas são de fundamental importância em redes: rede $\mathrm{e}$ centralidade do agente, subgrupos da rede, relações interorganizacionais.

Um ator é entendido como um indivíduo ou um grupo coletivo. A coletividade, ou a comunidade, promove o seu auto interesse, conferindo status relativamente mais elevados sobre os atores individuais que possuem recursos mais valiosos. ${ }^{24}$

$\mathrm{Na}$ centralidade do agente, percebeu-se diferenças de influência entre o agente mais central e o menos central, que aumentavam com a crescente hierarquia das estruturas.

A centralidade na rede é uma forma de quantificar o destaque de um ator ou grupo na rede. Centralidade do ator é aquele que está envolvido de forma intensa em relacionamentos com outros atores, seja como transmissor ou como receptor. Este envolvimento o torna mais visível em relação aos demais. ${ }^{25}$

$\mathrm{Na}$ modalidade de centralidade de grau ("degree centrality"), a centralidade do nó na rede está relacionada ao grau do nó. Nesta métrica, a visibilidade do nó na rede é o foco. O nó mais central é aquele que possui o maior grau. E esse nó central que está em contato direto com muito outros nós e ocupa um lugar de centralidade na rede. Em contrapartida, nós com grau menor ocupam uma posição periférica na rede..$^{25}$

Os dados podem ser relacionais, expressando uma relação entre os atores, que são representados por laços. ${ }^{25}$ Os laços apresentam uma relação de força, sendo forte ou fraca. ${ }^{26}$ Essa relação origina a densidade. A densidade em rede social é a relação das ligações efetivamente existentes entre os atores e o total de ligações possíveis entre esses atores. A densidade representa um índice do potencial de relação entre as partes da rede social. Essa representação condiz à quantidade e tipo de informação que podem ser trocados entre seus os componentes da rede.

Mordocco e Oliva $^{27}$ afirmam que a densidade refere-se ao nível de contatos dentro de uma rede, sendo que a densidade alta significa alto fluxo de comunicação na rede sem intermediários, ou seja, todos os integrantes da rede têm acesso direto a outro. Na densidade baixa há maior probabilidade de que as pessoas não estejam interligadas ou, somente estão interligáveis através de terceiros.

\section{MATERIAL E MÉTODOS}

A construção do corpus documental ocorreu por meio dos periódicos da base de dados Scielo Brasil (www.scielo.br), em que foi levantado o número de artigos publicados em cada periódico no período compreendido entre 2000 e 2011 . A coleta de dados ocorreu entre junho e setembro de 2013, e foi realizada na Universidade Tecnológica Federal 
do Paraná (UTFPR) do município de Ponta Grossa, localizado no estado do Paraná.

$\mathrm{Na}$ base de dados Scielo Brasil selecionou-se a guia "índice de assuntos". Para a busca do assunto, foi utilizado o unitermo "qualidade de vida" e em seguida a função "pesquisa". Os critérios de exclusão utilizados para seleção de artigos foram: duplicatas; os artigos em que se constatou que apesar de conter "qualidade de vida" no título, resumo ou palavraschave, versavam sobre outra temática. Os critérios de inclusão foram: Ter sido publicado no período entre 2000 e 2011; Ter sido publicado em periódicos.

Como resultado foi obtido uma listagem com todos os artigos relacionados ao termo. Realizou-se uma base de dados em planilha do Microsoft Excel com os artigos listados no resultado da busca. O processo foi repetido para todos os unitermos referentes à qualidade de vida constantes na base de dados Scielo: (i) Qualidade de Vida (QV); (ii) QV; (iii) QV e sustentabilidade; (iv) Qualidade de Vida Relacionada à Saúde (QVRS); (v) Qualidade de Vida no Trabalho (QVT); (vi) QVT; (vii) QVRS; (viii) QV relacionada ao estado de saúde; (ix) QVRS.

A formação da base de dados dessa pesquisa no Microsoft Excel, composta por artigos advindos do unitermo qualidade de vida e suas variações, contemplou as seguintes informações: (i) título do artigo; (ii) autores; (iii) unitermo que o artigo foi cadastrado na base de dados Scielo; (iv) Filiação institucional; (v) periódico da publicação; (vi) ano da publicação; (vii) área de atuação dos pesquisadores; (viii) área de conhecimento do periódico da publicação.

Utilizou-se os softwares Pajek e Ucinet na formação redes de autoria baseado nos métodos de redes sociais. Foram utilizados os procedimentos analíticos de centralidade (ou degree); main component e densidade. Tais procedimentos auxiliaram a identificar os componentes essenciais da rede para a consecução do objetivo o qual a pesquisa se propôs.

\section{RESULTADOS}

Considerando os artigos publicados de 2000 a 2011 na base de dados Scielo, o número de autores totaliza 2.529. Estes pesquisadores estão afiliados a 328 instituições distintas, das quais a maioria são Instituições de Ensino Superior (IES), hospitais, centros de pesquisa, clínicas ou instituições parceiras ao setor de pós-graduação, pesquisa e extensão. No período, totalizou-se 3.192 publicações em qualidade de vida em 100 periódicos distintos. Dos 3.192 artigos publicados de 2000 a 2011 na base de dados Scielo relativos ao tema de qualidade de vida, 3.152 foram indexados com o unitermo "QV", 21 foram indexados com o unitermo "QVRS" e 19 foram indexados com o unitermo "QVT".

Para a consecução do objetivo desta pesquisa foram identificadas as áreas de conhecimento dos pesquisadores que publicam no tema $\mathrm{QV}$ e suas respectivas variações, a área do conhecimento dos periódicos que abarcam as publicações em QV, as instituições que retêm os autores das publicações da área e as interações entre os autores que publicam no tema QV.

A Figura 1 apresenta o resultado obtido para a rede relativa à área de conhecimento dos periódicos, sendo que o tamanho do nó está representado de acordo com o cálculo de centralidade degree (número interações recebidas pela área do periódico). Os pesquisadores que publicaram seus artigos na base de dados Scielo Brasil entre 2000 e 2011 publicaram em periódicos de 44 áreas de conhecimento distintas, dentre as quais, destacam-se: medicina, psicologia, enfermagem, educação física, engenharia de produção, estatística e administração.

A Figura 2 mostra a rede por área de conhecimento que os pesquisadores pertencem. Os pesquisadores que publicaram em qualidade de vida na base de dados Scielo entre 2000 e 2011 foram agrupados em 76 áreas do conhecimento distintas, sendo que realizaram 254 interações (laços).

Foi realizado o procedimento de cutpoints para a rede instituições, para identificar as instituições que caso retiradas da rede desfragmentam significativamente parte das interações. A Figura 3 identifica as instituições que desfragmentam significativamente a rede (cutpoints). Por meio do procedimento de cutpoints estabeleceu-se instituições que representam pontos de desfragmentação significativos da rede. 
Revista de Ensino, Ciência e Inovação em Saúde v.2, n.1 (2021) 88-100

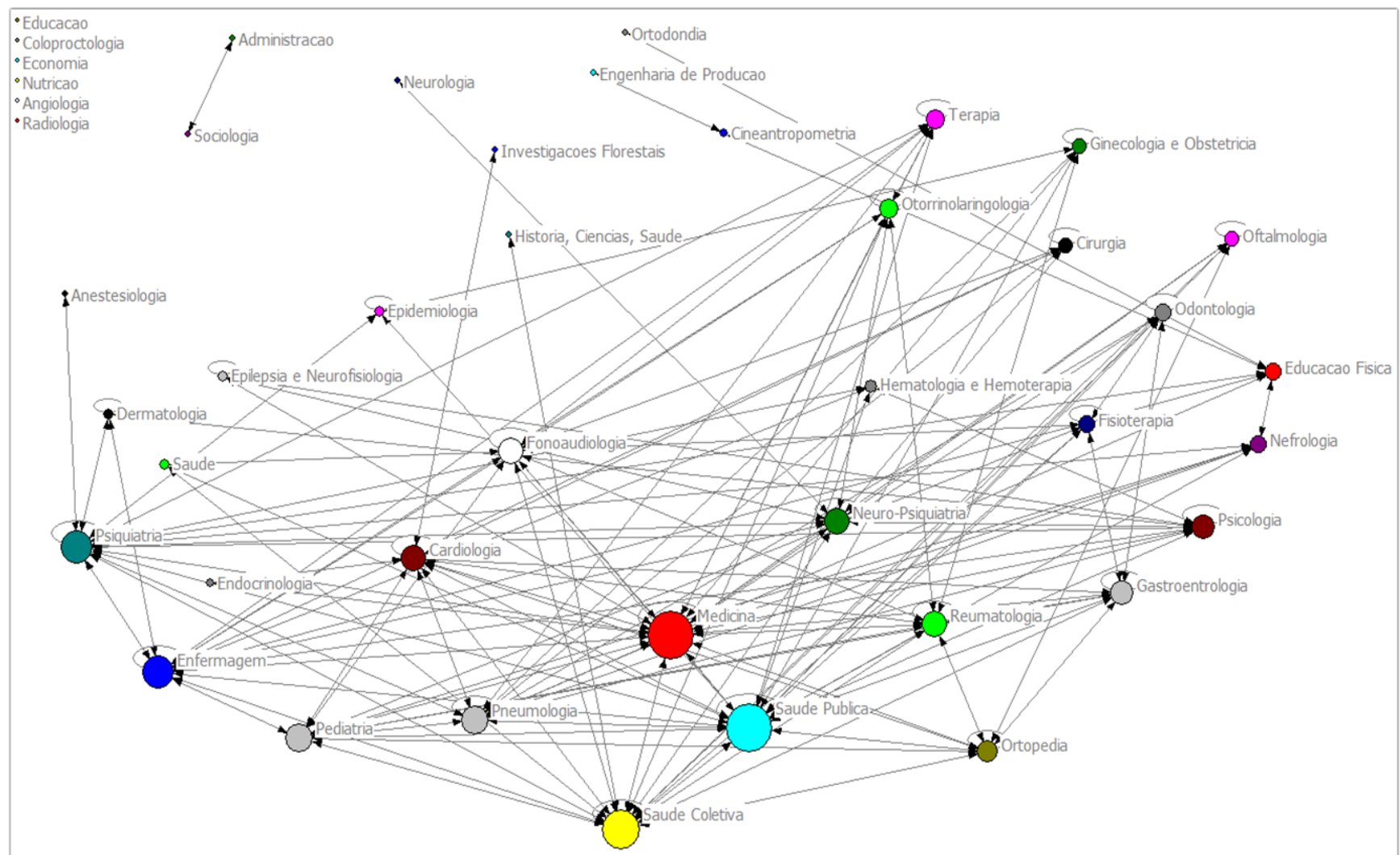

Figura 1. Rede área de conhecimento dos periódicos, julho a setembro, 2013, UTFPR - Ponta Grossa, Brasil.

- Imunologia e Alergia
- Biologia
- Ortodontia
- Sociologia
- Analista de dados
- Ciencias da comunicacao
- Jornalismo
- Endocrinologia
- Psicopatologia
- Economia
- Servico Social
- Musica
- Engenharia Civil
- Engenharia Agronomica
- Agronomia
- Ciencias Morfologicas
- Bioetica
- Radiologia
- Angiologia
- Traumatologia
- Hematologia
- Infectologista
- Obstetricia
- Medicina Social
- Obstetria
- Ergonomia
- Administracao
- Biociencias
- Patologia

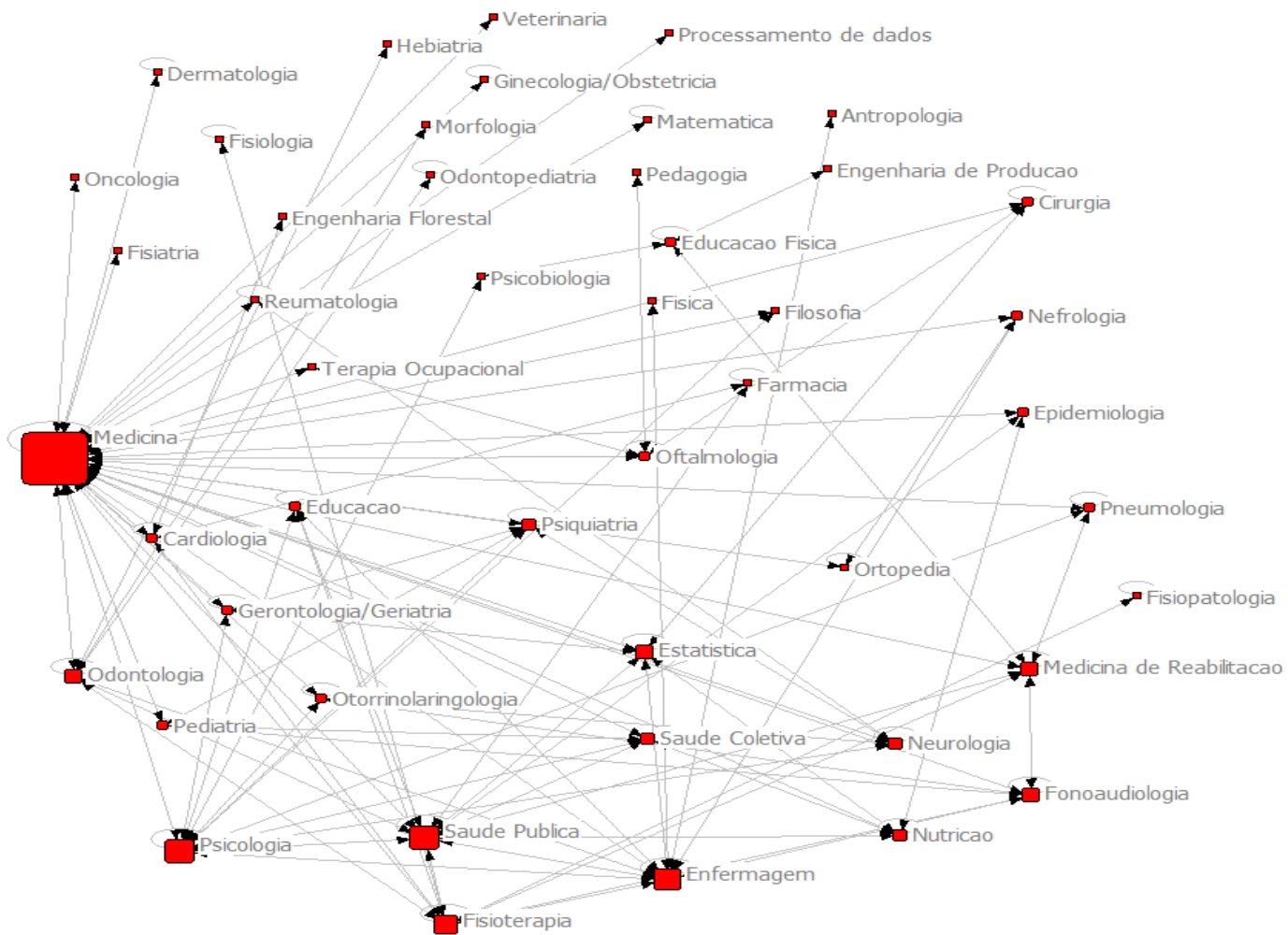

Figura 2. Rede área de conhecimento dos pesquisadores, julho a setembro, 2013, UTFPR - Ponta Grossa, Brasil. 


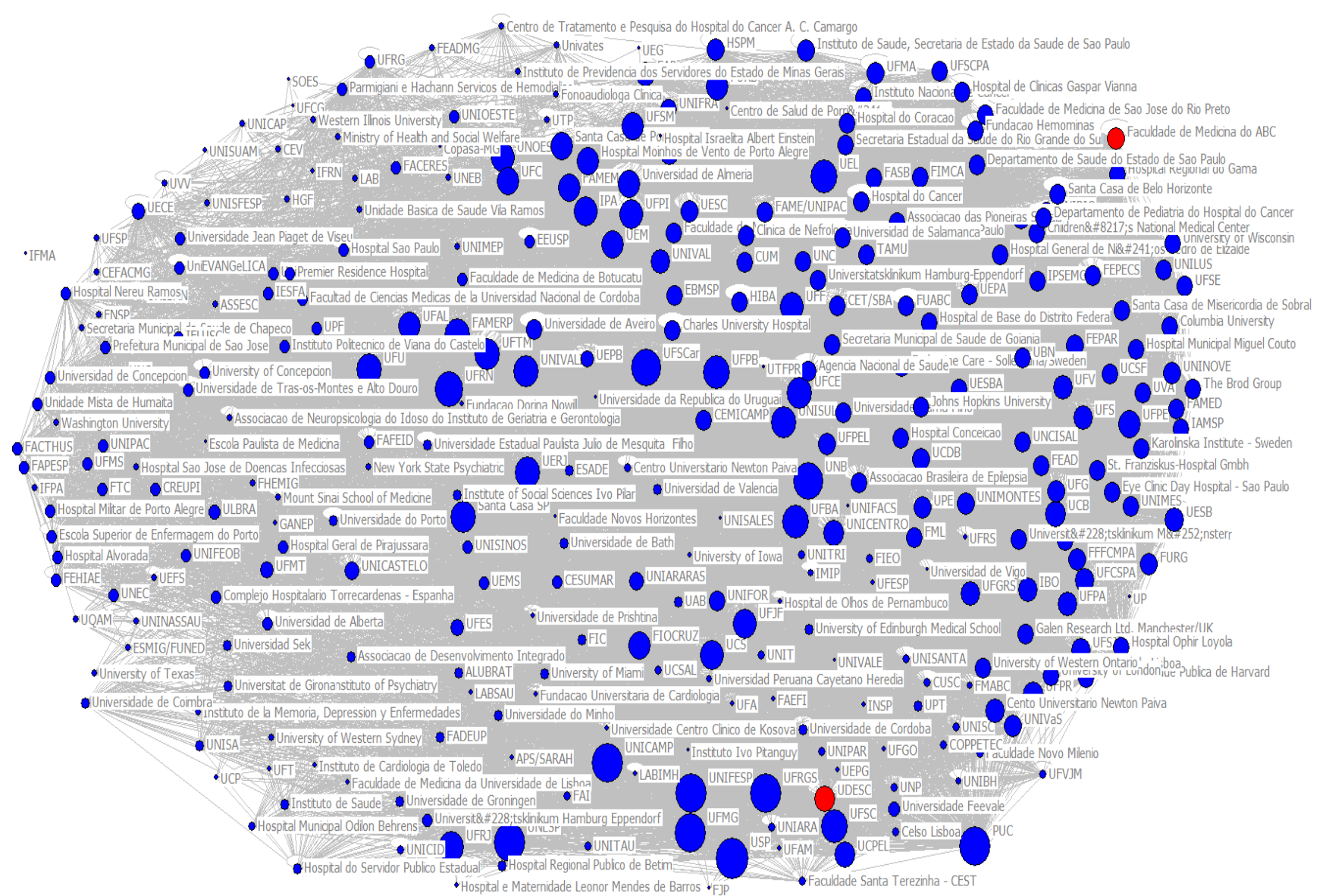

Figura 3. Cutpoints da rede instituições, julho a setembro, 2013, UTFPR - Ponta Grossa, Brasil.

Esses pontos foram detectados em duas das 328 instituições: Faculdade de Medicina do ABC e Universidade do Estado de Santa Catarina (UDESC). Retiradas da rede, essas duas IES reduzem o número de conexões existentes entre pesquisadores e demais instituições em 668 interações, pois a Faculdade de Medicina do ABC é responsável por 316 laços e a UDESC é responsável por 352 laços da rede.

Verificou-se, também, as instituições com maior contato direto com as demais instituições. A Figura 4 apresenta a centralidade de grau (degree centrality) para a rede de instituições com mais de 150 laços. O nó mais central é aquele que possui o maior grau. E esse nó central que está em contato direto com outros nós (no caso, instituições) e ocupa um lugar de centralidade na rede ${ }^{25}$.

Das 328 instituições, 65 apresentaram mais que 150 laços. O degree mostra a centralidade de um ator (instituição) tem, sendo os laços recebidos (indegree) ou enviados (outdegree). A Tabela 1 mostra o a centralidade de degree da rede de instituições para os nós com maior representatividade na rede. O InDegree representa o número de laços que o nó recebeu, enquanto que o OutDegree indica o número de laços que saíram do nó. A Tabela 2 mostra a densidade de cada rede e a centralidade de grau (InDegree e OutDegree) para a rede de autores. A lista contém os 20 autores com maior centralidade de grau. De forma geral, os pesquisadores com maior número de interações e/ou publicações na rede de autores ou maior centralidade de grau (degree), realizaram publicações com poucos pesquisadores (até três autores). Destacaram-se duas redes (separadas na cor rosa e alaranjado) de pesquisadores que apresentaram mais pesquisadores que publicaram em conjunto nos artigos indexados à base de dados Scielo relativos à qualidade de vida entre 2000 e 2011. É comum constatar um conjunto maior de pesquisadores atuando em um mesmo projeto de pesquisa quando esses projetos requerem uma coleta e análise de dados extensa. 


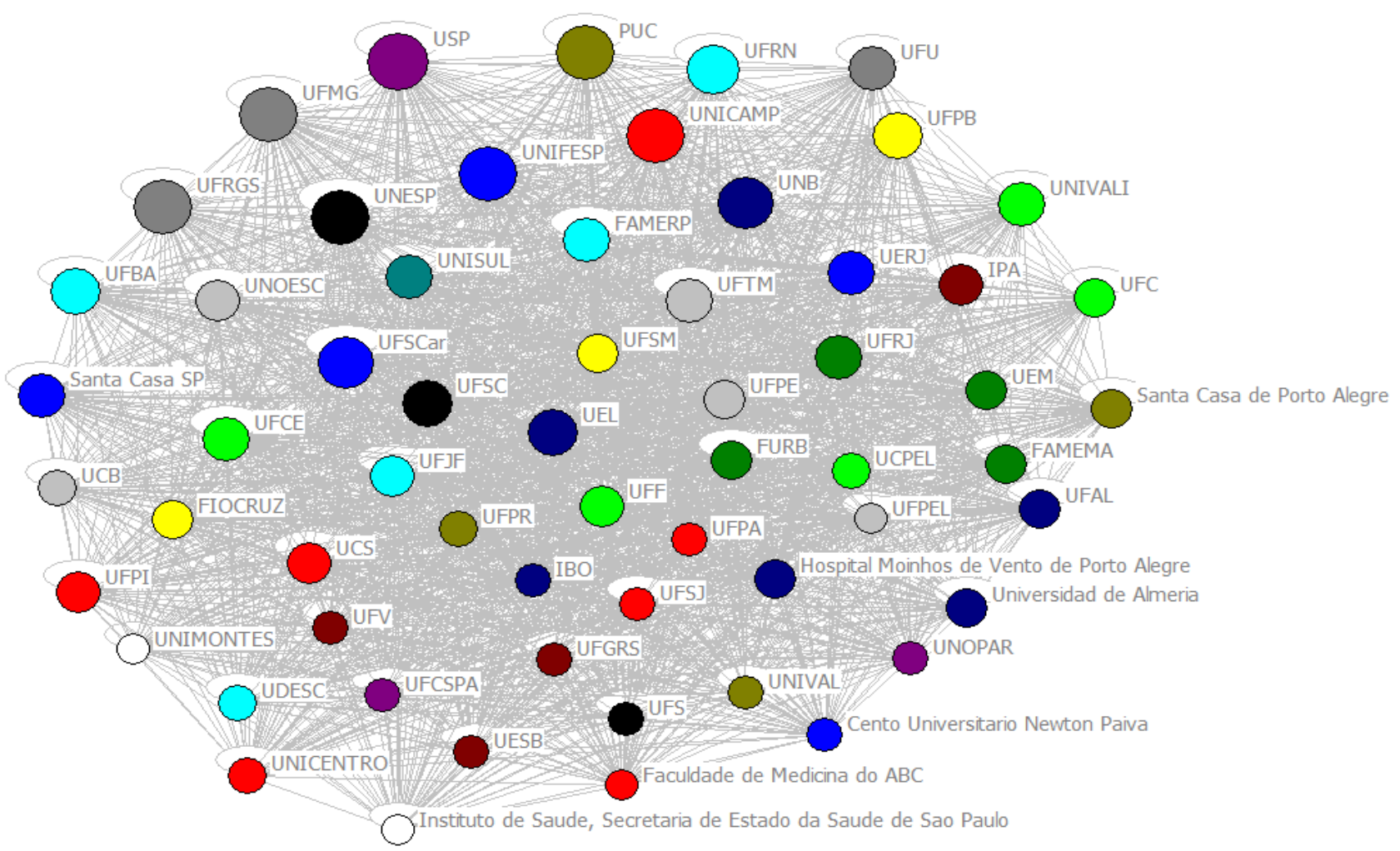

Figura 4. Rede instituições com mais de 150 laços, julho a setembro, 2013, UTFPR - Ponta Grossa, Brasil.

Tabela 1: Centralidade degree da rede instituições, julho a setembro, 2013, UTFPR - Ponta Grossa, Brasil.

\begin{tabular}{lcc}
\hline \multicolumn{1}{c}{ Instituição } & OutDegree & InDegree \\
\hline Universidade de São Paulo (USP) & 304.000 & 304.000 \\
Universidade Estadual de Campinas (UNICAMP) & 293.000 & 293.000 \\
Universidade Federal de Minas Gerais (UFMG) & 292.000 & 292.000 \\
Pontifícia Universidade Católica (PUC) & 291.000 & 291.000 \\
Universidade Federal de São Paulo (UNIFESP) & 289.000 & 289.000 \\
Universidade Federal do Rio Grande do Sul (UFRGS) & 284.000 & 284.000 \\
Universidade Estadual Paulista (UNESP) & 283.000 & 283.000 \\
Universidade de Brasília (UNB) & 271.000 & 271.000 \\
Universidade Federal de São Carlos (UFSCar) & 268.000 & 268.000 \\
Universidade Federal do Rio Grande do Norte (UFRN) & 261.000 & 261.000 \\
Universidade Federal de Santa Catarina (UFSC) & 244.000 & 244.000 \\
Universidade Federal da Paraíba (UFPB) & 242.000 & 242.000 \\
Universidade Federal da Bahia (UFBA) & 242.000 & 242.000 \\
Universidade Estadual de Londrina (UEL) & 239.000 & 239.000 \\
Universidade Federal do Rio de Janeiro (UFRJ) & 229.000 & 229.000 \\
Universidade do Sul de Santa Catarina (UNISUL) & 229.000 & 229.000 \\
Universidade Federal do Ceará (UFCE) & 229.000 & 229.000 \\
Universidade Federal de Uberlândia (UFU) & 226.000 & 226.000 \\
Universidade do Estado do Rio de Janeiro (UERJ) & 225.000 & 225.000 \\
Santa Casa de SP & 225.000 & 225.000
\end{tabular}


Revista de Ensino, Ciência e Inovação em Saúde v.2, n.1 (2021) 88-100

\begin{tabular}{|c|c|c|}
\hline Universidade Federal do Triângulo Mineiro (UFTM) & 225.000 & 225.000 \\
\hline Universidade do Vale do Itajaí (UNIVALI) & 223.000 & 223.000 \\
\hline Faculdade de Medicina de São José do Rio Preto (FAMERP) & 221.000 & 221.000 \\
\hline Universidade Federal Fluminense (UFF) & 218.000 & 218.000 \\
\hline Centro Universitário Metodista (IPA) & 216.000 & 216.000 \\
\hline Universidade Federal de Juiz de Fora (UFJF) & 214.000 & 214.000 \\
\hline Universidade do Oeste de Santa Catarina (UNOESC) & 210.000 & 210.000 \\
\hline Universidade de Caxias do Sul (UCS) & 209.000 & 209.000 \\
\hline Universidade Federal do Piauí (UFPI) & 206.000 & 206.000 \\
\hline Universidade Federal do Ceará (UFC) & 204.000 & 204.000 \\
\hline Universidade Federal de Alagoas (UFAL) & 203.000 & 203.000 \\
\hline Universidade Estadual de Maringá (UEM) & 199.000 & 199.000 \\
\hline Fundação Oswaldo Cruz (FIOCRUZ) & 197.000 & 197.000 \\
\hline Universidade Federal de Santa Maria (UFSM) & 196.000 & 196.000 \\
\hline Hospital Moinhos de Vento de Porto Alegre & 193.000 & 193.000 \\
\hline Universidade Regional de Blumenau (FURB) & 193.000 & 193.000 \\
\hline Universidade Federal de Pernambuco (UFPE) & 193.000 & 193.000 \\
\hline Faculdade de Medicina de Marília (FAMEMA) & 193.000 & 193.000 \\
\hline Universidad de Almeria & 193.000 & 193.000 \\
\hline Santa Casa de Porto Alegre & 193.000 & 193.000 \\
\hline Universidade Federal do Paraná (UFPR) & 187.000 & 187.000 \\
\hline Universidade Católica de Brasília (UCB) & 186.000 & 186.000 \\
\hline Universidade do Centro Oeste (UNICENTRO) & 183.000 & 183.000 \\
\hline Universidade Católica de Pelotas (UCPEL) & 181.000 & 181.000 \\
\hline Universidade do Estado de Santa Catarina (UDESC) & 175.000 & 175.000 \\
\hline Universidade Federal de São João Del-rei (UFSJ) & 173.000 & 173.000 \\
\hline Universidade Federal de Sergipe (UFS) & 173.000 & 173.000 \\
\hline Universidade Federal do Pará (UFPA) & 173.000 & 173.000 \\
\hline Universidade Federal de Ciências da Saúde de Porto Alegre (UFCSPA) & 172.000 & 172.000 \\
\hline Universidade do vale do Itajaí (UNIVAL) & 167.000 & 167.000 \\
\hline Cento Universitário Newton Paiva & 167.000 & 167.000 \\
\hline Instituto Brasileiro de Osteopatia (IBO) & 167.000 & 167.000 \\
\hline Universidade Estadual do Sudoeste da Bahia (UESB) & 167.000 & 167.000 \\
\hline Universidade Federal do Rio Grande do Sul (UFGRS) & 165.000 & 165.000 \\
\hline Universidade Federal de Viçosa (UFV) & 165.000 & 165.000 \\
\hline Universidade Estadual do Paraná (FAP) & 162.000 & 162.000 \\
\hline Universidade do Norte do Paraná (UNOPAR) & 162.000 & 162.000 \\
\hline Universidade de Pernambuco (UPE) & 159.000 & 159.000 \\
\hline Faculdade de Medicina do ABC & 157.000 & 157.000 \\
\hline Instituto de Saúde (Secretaria de Estado da Saúde de São Paulo) & 156.000 & 156.000 \\
\hline Universidade Federal de Pelotas (UFPEL) & 155.000 & 155.000 \\
\hline Universidade Católica Dom Bosco (UCDB) & 154.000 & 154.000 \\
\hline Universidade Federal de Goiás (UFG) & 153.000 & 153.000 \\
\hline Universidade do Vale do Sapucaí (UNIVAS) & 152.000 & 152.000 \\
\hline Universidade Estadual Montes Claros (UNIMONTES) & 151.000 & 151.000 \\
\hline
\end{tabular}

Fonte: Autoria própria

No entanto, o objetivo desta pesquisa não se ateve em descobrir a profundidade dos projetos de pesquisa de cada autor.

$\mathrm{O}$ resultado de centralidade degree mostra que os 20 autores com maior degree apresentam entre 36 e 14 laços de relacionamento cada um. $\mathrm{O}$
OutDegree e o InDegree foi representado pelo mesmo número de laços para todos os pesquisadores.

A densidade indica o potencial das relações entre os atores da rede social, mensurando as informações que podem ser trocadas nessa rede. Todas as redes apresentaram densidade baixa. As 
redes referentes à área de conhecimento dos pesquisadores e área de conhecimento dos periódicos apresentaram densidade extremamente baixa, significando que há maior probabilidade de as redes estarem interligadas somente por meio de terceiros (pesquisadores intermediários).

A rede de autores teve densidade baixa, porém, indica fluxo de comunicação na rede social com menor número de intermediários, em relação às demais redes.

\section{DISCUSSÃO}

Acerca dos resultados expostos na Figura 1, observa-se que os periódicos nas áreas de medicina, saúde pública e saúde coletiva abarcaram o maior número de publicações na área de QV (e derivações).

A área de engenharia de produção apresenta interações com educação física e cineantropometria, caracterizando um grupo realizado por um programa de pós-graduação específico com linha de pesquisa em QV.

Tabela 2: Centralidade de grau (degree) da rede autores, julho a setembro, 2013, UTFPR - Ponta Grossa, Brasil.

\begin{tabular}{|c|c|c|c|c|c|c|}
\hline Pesquisador & OutDegree & InDegree & Rede & Densidade & $\begin{array}{c}\text { Número } \\
\text { de } \\
\text { Laços } \\
\end{array}$ & $\begin{array}{c}\text { Degree } \\
\text { da } \\
\text { Rede } \\
\end{array}$ \\
\hline Rozana Mesquita & 36 & 36 & \multirow{4}{*}{$\begin{array}{c}\text { Rede de instituições } \\
\text { Rede de autores } \\
\text { Rede área de conhecimento } \\
\text { dos pesquisadores } \\
\text { Rede área de conhecimento } \\
\text { dos periódicos }\end{array}$} & 0,137 & 260 & 5,909 \\
\hline Lydia Masako & 27 & 27 & & 0,312 & 33.482 & 102,079 \\
\hline $\begin{array}{l}\text { Marcelo Pio de } \\
\text { Almeida Fleck }\end{array}$ & 26 & 26 & & 0,033 & 190 & 2,500 \\
\hline Rubens Belfort & 22 & 22 & & 0,002 & 10.804 & 4,272 \\
\hline $\begin{array}{l}\text { Daniela } \\
\text { Francescato }\end{array}$ & 21 & 21 & & & & \\
\hline Yara Juliano & 19 & 19 & & & & \\
\hline Fernando Freitas & 19 & 19 & & & & \\
\hline Rosana Aparecida & 18 & 18 & & & & \\
\hline Sirlei Siani & 17 & 17 & & & & \\
\hline $\begin{array}{l}\text { Luiz Antonio } \\
\text { Nogueira-Martins }\end{array}$ & 16 & 16 & & & & \\
\hline Auro Del Giglio & 16 & 16 & & & & \\
\hline $\begin{array}{l}\text { Claudia Benedita } \\
\text { dos Santos }\end{array}$ & 16 & 16 & & & & \\
\hline $\begin{array}{l}\text { Eduardo } \\
\text { Chachamovich }\end{array}$ & 16 & 16 & & & & \\
\hline $\begin{array}{l}\text { Marcos Bosi } \\
\text { Ferraz }\end{array}$ & 16 & 16 & & & & \\
\hline $\begin{array}{l}\text { Jose Roberto } \\
\text { Jardim }\end{array}$ & 15 & 15 & & & & \\
\hline $\begin{array}{l}\text { Aarão Mendes } \\
\text { Pinto Neto }\end{array}$ & 15 & 15 & & & & \\
\hline $\begin{array}{l}\text { Elza Marcia } \\
\text { Targas Yacubian }\end{array}$ & 14 & 14 & & & & \\
\hline $\begin{array}{l}\text { Anamaria Siriani } \\
\text { de Oliveira }\end{array}$ & 14 & 14 & & & & \\
\hline Jamil Natour & 14 & 14 & & & & \\
\hline $\begin{array}{l}\text { Neide Barreira } \\
\text { Alonso }\end{array}$ & 14 & 14 & & & & \\
\hline
\end{tabular}

Fonte: Autoria própria 
As publicações em QV não possuem como foco final os periódicos da área de administração ou sociologia, que apresentaram interação. Sugere-se que os pesquisadores da área de Ciências Sociais Aplicadas tenham desviado o foco do estudo de QV, não realizando publicações em periódicos da área. Além disso, é possível perceber diversas áreas da saúde isoladas e uma área relacionada às ciências exatas (economia).

Conforme Faust e Wasserman ${ }^{25}$ apontam, sendo os dados relacionais, os laços expressam uma interação entre os atores. A rede das áreas de conhecimento dos periódicos é formada por 306 laços ou interações entre os pesquisadores que publicaram nas 44 áreas dos distintos periódicos. A área de medicina detém 44 laços, a área de saúde pública capta igualmente 44 e a área de saúde coletiva detém 36 laços ou interações. Cabe salientar que as três áreas realizam interações com pesquisadores de outras áreas e com pesquisadores da própria área.

Algumas áreas apresentam destaque em número de interações, mas não interagem ou realizam publicações na área de QV com pesquisadores das próprias áreas são odontologia, educação física, nefrologia e pediatria.

Os periódicos que receberam maior número de publicações foram Arquivos de Neuro-Psiquiatria (182 publicações), Cadernos de Saúde Pública (151 publicações), Ciência \& Saúde Coletiva (133 publicações), Jornal Brasileiro de Pneumologia (133 publicações) e Revista de Saúde Pública (116 publicações). Esses cinco periódicos captaram $22,4 \%$ do total das publicações do período.

Acerca dos resultados expostos na Figura 2, assim como ocorreu na identificação da área de conhecimento dos periódicos que abarcaram as publicações em QV da base de dados Scielo de 2000 a 2011, a área de medicina e áreas correlatas, são as áreas dos pesquisadores que mais publicam em QV. Os pesquisadores formados em medicina que escrevem no tema QV são uma espécie de "centralizadores" ou pesquisadores formadores de grupos que publicam sobre $\mathrm{o}$ assunto. Esses pesquisadores interagem com pesquisadores de outras áreas e com pesquisadores da própria área. A área de medicina é responsável por 66 interações (laços da rede).

Diferentemente com o que ocorre com a grande área de medicina, os pesquisadores com formação em administração, biociências e na área específica de patologias (em medicina), os pesquisadores realizaram publicações em qualidade de vida (de 2000 a 2011) com pesquisadores de igual formação acadêmica.

Percebe-se que diversas áreas do conhecimento não possuem conexão com as demais. As áreas que não apresentam interações com as demais são representadas por áreas específicas de medicina (como imunologia e alergia, ciências morfológicas, angiologia, traumatologia, infectologia e patologia), administração, economia, ciências da comunicação, e algumas áreas das engenharias. Também é possível salientar que em uma análise detalhada, as áreas de análise de dados, processamento de dados, estatística, física e ergonomia representaram áreas complementares ou de suporte às demais áreas do conhecimento que realizam pesquisas em $\mathrm{QV}$.

Foram detectadas 328 instituições que afiliaram os pesquisadores que realizaram publicações em qualidade de vida indexadas à base de dados Scielo entre 2000 e 2011. Das 328 instituições, apenas os pesquisadores do Instituto Federal do Maranhão (IFMA) não realizaram interações com as demais IES, ou seja, 327 instituições formam uma grande rede de interações, composta por 33.786 laços, o main component da rede de instituições. $\mathrm{O}$ resultado do procedimento main component enfatiza uma rede coesa e com muitas ligações entre todos os membros (instituições), dificultando a extração de um único componente (instituição) principal.

A USP destacou-se como a instituição com maior número de ligações com outras instituições de ensino superior. Este fato pode justificar-se pelo pioneirismo da USP em programas de pósgraduação, tal qual aponta Nascimento et al. ${ }^{27}$ (2009) para a área da contabilidade. Em seguida destaca-se a UNICAMP e a UFMG.

Logo, entre as primeiras colocadas tem-se instituições públicas e da região sudeste. Contexto semelhante foi evidenciado por Allegretti et al. ${ }^{17}$ na área de administração pública em saúde. Este cenário está relacionado com as características da produção científica no Brasil, que está diretamente ligada aos programas de pós-graduação Stricto Sensu, que estão localizados em maior proporção em instituições da região sul e sudeste ${ }^{1,28,29,30,31}$.

As instituições possuem origem nacional e internacional, sendo que as internacionais são de países como Alemanha, Espanha, Suécia, Estados Unidos, Argentina, Peru, Portugal, Austrália, República da Sérvia, entre outros. A maioria das instituições são IES, sendo as demais são institutos 
de pesquisa, centros clínicos, hospitais especializados e secretarias de saúde.

Sobre os resultados expostos nas Figura 3 e Figura 4, e na tabela 1, para Faust e Wasserman ${ }^{25}$ a centralidade na rede indica o ator que está envolvido de forma intensa em relacionamentos com outros atores, seja como transmissor ou como receptor. $\mathrm{Na}$ modalidade de centralidade de grau ("degree centrality"), o nó mais central é aquele que possui o maior grau. Tomando como ponto de partida a teoria de Faust e Wasserman ${ }^{25}$, as instituições das 65 instituições com mais que 150 interações, a Universidade de São Paulo (USP) apresentou o maior InDegree e OutDegree, seguida da Unicamp e UFMG, indicando que as instituições possuem prestígio, sociabilidade e centralidade na rede de publicações no tema de QV em periódicos indexados à base de dados Scielo.

Dos 2.529 autores que publicaram seus artigos em QV entre 2000 e 2011 na base de dados Scielo, poucos foram os autores que apresentam uma produção científica totalmente independente, sem parcerias com os demais pesquisadores. A maioria dos autores trabalha em redes de coautoria.

Os 2.529 autores formaram uma rede com 10.894 laços. Desse total, 603 interações foram publicações de um artigo entre (i) um, (ii) dois ou (iii) três autores. Desse total, 26 autores realizaram publicações individuais no tema de QV em periódicos indexados à base de dados Scielo entre 2000 e 2011, 199 autores publicaram em dupla e 378 autores publicaram artigos em trios.

A utilização das redes não trata-se de uma anormalidade. $\mathrm{O}$ estabelecimento de parcerias entre autores, ou mesmo colaborações entre instituições/órgãos facilitam a partilha de recursos e informações necessárias para a realização dos estudos. Estes mecanismos, por sua vez, elevam a qualidade das pesquisas e a probabilidade de que os resultados sejam eficazes ${ }^{32}$.

Os autores que apresentaram maior degree foram Rozana Mesquita (36), Lydia Masako (27) e Marcelo Pio de Almeida Fleck (26). O degree indica o número de laços que um pesquisador tem com outros pesquisadores dentro de uma rede. Em outras palavras, um maior degree representa que determinado autor tem maior importância sobre os demais, que acabam dependendo dele no processo de troca de informações ${ }^{33}$. Neste sentido, é possível indicar que os autores supracitados acabam sendo centrais nas pesquisas sobre qualidade de vida, liderando grupos de pesquisa com elevado número de publicações.
Além disso, há a possibilidade de que estes autores sejam bastante procurados para o estabelecimento de parcerias. Riviera indica que um dos fatores que aumentam a possibilidade de que os estudos sejam divulgados ou citados pela comunidade científica é a inclusão de autores que já são altamente $\operatorname{citados}^{34}$. Neste sentido, é natural que os pesquisadores que se destacam, publicam mais e são bastante citados sejam procurados para pesquisas em conjunto, tendo em vista a possibilidade de melhorar a imagem da comunidade científica ante um determinado estudo. Cenário semelhante, com evidência de que as redes se configuram no entorno de professores mais produtivos, foi apontado por Maia e Caregnato $^{13}$ em uma investigação sobre indicadores de co-autoria (2008).

No tocante a tabela 2 o resultado encontrado nas publicações no tema $\mathrm{QV}$ indexadas à base de dados do Scielo entre 2000 e 2011 não é coerente com os modelos clássicos de QVT apresentados por Pedroso ${ }^{35}$, sendo eles o de Walton (1973), Hackman e Oldham (1974), Westley (1979), Werther e Davis (1981) e Nadler e Lawler (1983).

Uma das possíveis justificativas é devido ao número de artigos referentes somente à QVT ser pequeno na base Scielo. Já os artigos indexados com o unitermo QV são predominantes na base e são estudados a partir do instrumento de avaliação da QV WHOQOL $^{36}$, desenvolvido pela Organização Mundial da Saúde.

A QV era estudada a partir de dois focos: pessoas doentes ou pessoas escravizadas no trabalho. Pessoas que fugiam ao padrão aceitável da sociedade (ditas "normais") não era foco dos estudos. Esse fator pode ter auxiliado para a descentralização das áreas de conhecimento que estudam QV.

\section{CONCLUSÃO}

Com a pesquisa desenvolvida, são possíveis as seguintes inferências em relação ao objetivo e hipótese do presente estudo:

1. Comparar as publicações dos pesquisadores das diversas áreas do conhecimento que realizam pesquisas em QV indexadas à base de dados Scielo: os pesquisadores de medicina realizam maior número de publicações.

2. Analisar a concentração das áreas do conhecimento que publicam em qualidade de vida indexadas à base de dados Scielo: percebese predominância da área de medicina e áreas da saúde de forma geral. 
3. Analisar a concentração das instituições que realizaram maior número de publicações em qualidade de vida indexadas à base de dados Scielo: as instituições USP, Unicamp e UFMG são instituições centrais na rede, possuindo a maior centralidade de grau.

4. Delimitar a área de concentração dos periódicos que tem maior número de publicações em QV indexadas à base de dados Scielo: a área da saúde, principalmente medicina, saúde pública e saúde coletiva concentram os periódicos com maior número de publicações.

Percebe-se uma descentralização dos estudos do tema QV e QVT, cujas teorias clássicas foram propostas principalmente por sociólogos e administradores.

\section{REFERÊNCIAS}

1. Dias TMR, Moita GF, Dias PM. Um retrato da produção científica brasileira baseado em dados da plataforma Lattes. Brazillian Journal of Information Studies: Research Trends 2018; 12(4): 62-74. https://seer.ufrgs.br/EmQuestao/article/view/81536 2. Scarpelli AC et al. Academic trajectories of dental researchers receiving $\mathrm{CNPq}^{\prime}$ 's productivity grants. Brazilian Dental Journal 2008; 19(3): 252-256.

3. Macias-Chapula CA. O papel da informetria e da cienciometria e sua perspectiva nacional e internacional. Ciência da Informação 1998; 27(2): 134-140.

4. Silva JA, Bianchi MLP. Cientometria: a métrica da ciência. Paidéia 2001; 11(21): 5-10.

5. Teodorescu D. Correlates of faculty publication productivity: A cross-national analysis. Higher Education 2000; 39(2): 201-222.

6. Richard $\mathrm{CM}$ et al. Supporting academic publication: evaluation of a writing course combined with writers'support group. Nurse Education Today 2009; 29(5): 516-521.

7. Wendt GW et al. Perfil dos bolsistas de produtividade em pesquisa do CNPq em Psicologia. Psicologia: Ciência e profissão 2013; 33(3): 536547.

8. Beaver DB, Rosen R. Studies in scientific collaboration: part III: professionalization and the natural history of modern scientific co-authorship. Scientometrics 1979; 1(3): 231-245. https://doi.org/10.1007/BF02016308

9. Nascimento S, Beuren IM. Redes sociais na produção científica dos programas de pósgraduação de ciências contábeis. Revista de Administração contemporânea 2011; 15(1): 47-66.
10. Packer A. Entrevista Abel Packer. Scielo em Perspectiva [internet]. 2013 [citado 2013 jul 15]; 1(1):1-1. Disponível em: https://blog.scielo.org/blog/2013/07/15/entrevistaabel-packer/\#.YBHsaehKg2x

11. Meneghini R. É hora de mudança. Ciência hoje [internet]. 2012 [citado 2012 jul 2014]; 1(1):1-1. Disponível em: http://cienciahoje.uol.com.br/noticias/2012/09/ehora-de-mudanca. Acesso em: 06 jul. 2014.

12. Andrighi FF, Hoffmann VE, Andrade MAR. Análise da produção científica no campo de estudo das redes em periódicos nacionais e internacionais. Revista de administração e inovação 2011; 8(1): 2954. https://www.scielo.br/scielo.php?pid=S0034$76122018000400571 \&$ script $=$ sci_abstract $\&$ tlng=es

13. Maia MFS, Caregnato SE. Co-autoria como indicador de redes de colaboração científica. Perspectiva em ciência da informação 2008; 13(2): 18-31.

https://www.scielo.br/scielo.php?\%20pid=S141399362008000200003\&script=sci_arttext

14. Grácio MCC. Colaboração científica: indicadores relacionados de coautoria. Brazillian Journal of Information Studies: Research Trends 2018; 242(2): 22. https://revistas.marilia.unesp.br/index.php/bjis/articl e/view/7976

15. Firme SM, Miranda ACD, Silva JA. Produção do conhecimento científico: um estudo das redes colaborativas. Biblos: Revista do instituto de ciências humanas e da informação 2017; 31(2): 4561.

https://periodicos.furg.br/biblos/article/view/8019 16. Leite DBC, Caregnato CE, Miorando BS. Efeitos multiplicadores das redes de colaboração em pesquisa. Um estudo internacional. Avaliação: Revista da avaliação da Educação Superior 2018; 23(1): 263-286.

https://www.scielo.br/scielo.php?pid=S1414$40772018000100263 \&$ script $=$ sci_arttext\&tlng $=p t$ 17. Allegretti ACV et al. Redes sociais na produção científica em administração pública em saúde no Brasil. Revista de Administração Pública 2018; 52(4): 571-592.

https://www.scielo.br/scielo.php?pid=S0034$\underline{76122018000400571 \& \text { script }=\text { sci_abstract\&tlng }}=\mathrm{es}$ 18. Conner N, Provedel A, Maciel ELN. Ciência e saúde coletiva: análise da produção científica e redes colaborativas de pesquisa. Ciência e saúde coletiva 2017; 22(3): 1-1. https://www.scielosp.org/article/csc/2017.v22n3/98 7-996/pt/\# 
19. Guimarães IP. Produção científica em rede de colaboração no campo da política educacional no Brasil (2000-2014). Revista de estudios teóricos y epistemológicos en política educative 2018; 3(1): 138.

20 Mizruchi MS. Análise de redes sociais: avanços recentes e controvérsias atuais. Revista de Administração de Empresas 2006; 46(3): 72-86. http://dx.doi.org/10.1590/S0034-75902006000300013 21 Souza Q, Quandt C. Metodologia de análise de redes sociais. In: Duarte F, Quandt C, Souza Q. (editors). $\mathrm{O}$ tempo das redes. São Paulo: Perspectivas; 2008. p. 31-64.

22 Noody W, Mrvar A, Batagelj V. Attributes and relations. In: Noody W, Mrvar A, Batagelj V. (editors) Exploratory social network analysis with Pajek. New York: Cambridge University Press, 2005. p. 29-32.

23 Castells M. A sociedade em rede: a era da informação, economia, sociedade e cultura. São Paulo: Terra, 2002.

24 Lin N. Social capital: a theory of social structure and action. Cambridge: Cambridge University Press, 2001. Capítulo 1, Resources, hierarchy, networks, and homophily: the structural foundation; p. 29-40.

25 Faust K, Wasserman S. Centrality and Prestige: A Review and Synthesis. Journal of Quantitative Anthropology 1992; 4(1): 23-78.

https://archive.org/details/sim journal-ofquantitative-anthropology 199219934 1/page/n3/mode/2up

26. Granovetter M. The strength of weak ties. American. Journal of Sociology 1973; 78(6): 13601380.

https://www.jstor.org/stable/2776392?seq=1

27. Mordocco SO, Oliva EC. Análise de redes sociais informais em uma empresa de pequeno porte: um estudo do segmento de prestação de serviços. In: XI Seminário em administração; 2008 ago 28-29; São Paulo. São Paulo: Faculdade de Economia, Administração, Contabilidade e Atuária; 2008. P. 111. (Revista REGE-USP, 2008).
28. Coutinho RX et al. Brazilian Scientific production in Science Education. Scientometrics 2012; 92(3): 697-710.

29. Gripp C, Testi BM. Trajetórias acadêmicas: um estudo comparado da carreira acadêmica em Minas Gerais. Sociedade e Estado 2012; 27(1): 47-61.

30. Guimarães R. O futuro da pós-graduação: avaliando a avaliação. Revista Brasileira de PósGraduação 2011; 4(8): 282-292.

31. Klepa TC, Pedroso B. Análise da produção técnico-científica de bolsistas de produtividade do Conselho Nacional de Desenvolvimento Científico e Tecnológico (CNPq) na área da Pediatria. Revista Eisten 2019; 18(20): 1-6.

32. Bevc CA, Retrum JH, Varda DM. New perspectives on the "Silo Effect": Initial Comparisons of Network Structures Across Public Health Collaboratives. Am J Public Health 2015; 105 (2):

230-235.

https://www.ncbi.nlm.nih.gov/pmc/articles/PMC43 $\underline{55706 /}$

33. Rossoni L, Guarido Filho ER. Cooperação entre programas de pós-graduação em administração no Brasil: evidências estruturais em quatro áreas temáticas. Revista de Administração Contemporânea 2009; 13(3): 366-360. https://www.scielo.br/scielo.php?pid=S1415$65552009000300003 \&$ script $=$ sci_abstract\&tlng $=\mathrm{pt}$ 34. Riviera E. Scientific communities as autopoietic systems: the reproductive function of citations. Journal of the American Society for Information Science and Technology 2013; 64(7): 1442-1453. https://onlinelibrary.wiley.com/doi/abs/10.1002/asi. 22826

35. Pedroso B. Desenvolvimento do TQWL-42: um instrumento de avaliação da qualidade de vida no trabalho [dissertação]. Ponta Grossa (PR): Universidade Tecnológica Federal do Paraná, 2010. 36 Pedroso B. Desenvolvimento do TQWL-42: um instrumento de avaliação da qualidade de vida no trabalho [dissertação]. Ponta Grossa (PR): Universidade Tecnológica Federal do Paraná, 2010. 